www.nature.com/ijo

\title{
EDITORIAL
}

\section{Bariatric surgery highlights in the International Journal of Obesity}

International Journal of Obesity (2012) 36, 327; doi:10.1038/ijo.2011.230

This issue marks the first appearance of Bariatric Surgery Highlights in the International Journal of Obesity (IJO). Bariatric surgery originated in 1954 and publications in the field were published mainly in surgical journals for many years. Many of these articles were devoted to improving surgical techniques to enhance weight-loss results. In recent years, IJO has seen an upsurge in submissions of manuscripts on bariatric surgery, including many that delve into the physiological changes that occur with the various forms of bariatric surgery. It has long been apparent that bariatric surgery achieved better results in very obese patients than could be obtained by medical treatment. The recognition that bariatric surgery produced its effects through physiological changes rather than simple mechanical changes to the intestines or stomach was recognized in the 1970 s. In recent years, the contribution of altered gastrointestinal hormones increasingly has been recognized and the complexity of the changes after bariatric surgery appreciated. This raises the possibility that the mechanisms of weight loss with bariatric surgery may be sufficiently identified with further research, so that medical or pharmacological means of reproducing these effects could be developed. At the least, improved surgical techniques may be identified to enhance weight loss and reduce adverse effects.

IJO has made its mission to cover the field of obesity research as broadly as possible. The Editors and Editorial Board of IJO discussed the importance of bariatric surgery to the field of obesity and the desirability of bringing surgeons and other obesity practitioners closer together. The decision was made to encourage submission to IJO of manuscripts on bariatric surgery and to have two issues per year (February and October) in which the best of the bariatric surgery articles will be featured. Each Bariatric Surgery Highlights issue will contain four to eight papers on some aspect of bariatric surgery and one to two reviews on the topic. Other papers on bariatric surgery for which we do not have room, or for which the timing is not right, will be published during regular issues. We hope the increased attention by IJO to the field of bariatric surgery will bring new investigators, both surgeons and other scientists, to the field and that this will accelerate progress towards improving treatment of obesity and its complications.

Dr Philip Schauer, Professor of Surgery at Cleveland Clinic, Cleveland, Ohio, has been invited to assume the role of Associate Editor for Bariatric Surgery. Dr Schauer is a pioneer in the field and is Past President of the American Society for Metabolic and Bariatric Surgery. Dr Schauer and the other Editors of IJO encourage all investigators working in the area of bariatric surgery to submit their best manuscripts to IJO.

\section{RL Atkinson ${ }^{1}$ and I Macdonald ${ }^{2}$}

${ }^{1}$ Obetech Obesity Research Center, Richmond, VA, USA and ${ }^{2}$ School of Biomedical Sciences, University of Nottingham Medical School, Queen's Medical Centre,

Nottingham, UK E-mail: ratkinson2@vcu.edu 